\title{
Optical Communication Through Random Atmospheric Turbulence
}

\author{
Jean-Pierre Laussade, Amnon Yariv, and Jack Comly
}

\begin{abstract}
In this paper we compare the theoretical performances of two schemes of optical communication through the atmospheric turbulence: (1) heterodyne detection and (2) video detection. The signal-to-noise ratios $(S / N)$ in the output current of a detector are expressed for both schemes in terms of the correlation function of the refractive index fluctuations of the turbulence. The results of a separate theoretical analysis of optical wave propagation through a random turbulence are used in order to obtain a numerical estimate of the performance criterion $(S / N)_{(2)} /(S / N)_{(1)}$ in terms of the length of propagation through the atmosphere, the turbulence strength, the wavelength of the optical wave, and the diameter of the receiving aperture.
\end{abstract}

\section{Introduction}

In this paper we consider the effect of propagation through a turbulent atmosphere on two archetypes of optical communication schemes: (1) a superheterodyne and (2) a video communication system.

We are aiming our analysis specifically at electrooptic modulation systems which, fundamentally, control the phase of the optical carrier. This modulation is often converted into amplitude modulation by means of polarizers, or recovered as phase modulated intermediate frequency signal in a heterodyne detection system. Since our main interest in this analysis is in the loss of signal information due to random phase fluctuations and not in the many possible detection schemes, we define, somewhat arbitrarily, our signal power as that of the modulation frequency sidebands in the output of the optical nonlinear detector. In the case of the heterodyne system, the detector output, being phase modulated, requires further demodulation to recover the signal. It is assumed that this process does not change the signal-to-noise ratio materially, so that the comparison between the video and heterodyne schemes can be carried out at the same point of the communication link, i.e., at the output of the first (optical) detector.

To be specific, in scheme (1) the linearly polarized output field of a laser oscillating at $\omega_{0}$ is phase modulated at a frequency $\omega_{m}$. After propagating through a turbulent atmosphere, it is incident on the surface of a nonlinear optical detector along with a local oscillator signal ${ }^{1}$ whose frequency is $\omega_{0}-\Delta \omega$. For the purposes of this analysis, we define the signal as the components of the detector current oscillating at $\left(\Delta \omega \pm \omega_{m}\right)$.

The authors are with the California Institute of Technology, Pasadena, California 91109.

Received 6 January 1969.
In scheme (2), the video communication system, the phase modulation at $\omega_{m}$ is impressed on one of the two mutually orthogonal polarizations which initially add up to form the linearly polarized laser field. These two polarizations, having traversed together the turbulent path, are mixed in the nonlinear detector to produce an output current at $\omega_{m}$ to which we refer as the signal. From the point of view of signal-to-noise ratio, the best we can do is have the main source of noise in the output of the detector be the shot noise generated by the local oscillator in case (1) or the optical signal itself in case (2). ${ }^{2,3}$

The main purpose of this work is to apply the results of a theoretical analysis of propagation through random atmospheric turbulence ${ }^{4}$ to evaluate the signal-to-noise ratio following detection in the cases of a superheterodyne receiver and a video receiver as described above. The relative performance of these systems is calculated as a function of optical wavelength, the path length, the receiver diameter, and the strength of the turbulence.

\section{Signal-to-Noise Ratio in Optical Mixing Detection}

In the most general case, we assume that in the plane of the detector the reference and the information signals have random amplitudes and phases due to the random fluctuations of the refractive index along the propagation path in the atmosphere. At a point defined by the coordinates $(L, \mathrm{r})$ in the plane of the detector, the modulated optical electric field is

$$
E_{S}(L, \mathbf{r})=A_{S}(L, \mathbf{r}) \exp \left\{i\left[\omega_{0} t+\phi_{m}(t)+\phi_{s}(L, \mathbf{r})\right]\right\},
$$

where $L$ is the length of the communication link, $A_{S}(L,-$ r) are the random amplitude and phase due to the turbulent nature of the transmission medium, $\omega_{0}$ is the frequency of the optical wave, $\phi_{m}(t)$ is the modulation 
phase containing the information. We consider the case of harmonic phase modulation so that $\phi_{m}(t)=\delta$ $\sin \omega_{m}(t)$, where $\delta$ is the modulation index and $\omega_{m}$ is the (angular) modulation frequency.

The complex reference electric field is taken as

$$
E_{R}(L, \mathrm{r})=A_{R}(L, \mathrm{r}) \exp \left\{i\left[\left(\omega_{0}-\Delta \omega\right) t+\phi+\phi_{R}(L, \mathrm{r})\right]\right\},
$$

where $A_{R}(L, \mathbf{r})$ and $\phi_{R}(L, \mathbf{r})$ are the random amplitude and phase due to the turbulent transmission medium; $\phi$ is a constant phase factor; and $\Delta \omega$ is the frequency offset from the carrier frequency. (For video detection, $\Delta \omega=$ 0.) The total electric field in the plane of the detector is the sum of the information and reference electric fields:

$$
E_{T}(L, \mathrm{r})=E_{S}(L, \mathbf{r})+E_{R}(L, \mathrm{r}),
$$

and the output current of the nonlinear detector (after averaging over a few optical periods) is taken as

$$
i(t)=\frac{\eta q}{2 h \nu} \int_{\Sigma} d \mathrm{r} E_{T}(L, \mathrm{r}) E_{T} *(L, \mathrm{r}),
$$

where $\eta$ is the quantum efficiency of the detector, $\nu$ equals $\omega_{0} / 2 \pi, q$ is the electronic charge, and $\Sigma$ is the area of the detector.

The output current $i(t)$ has a dc part $i_{D C}$ and oscillating components at sideband frequencies $\Delta \omega \pm \omega_{m}$, where $m= \pm 1, \pm 2, \ldots$ Here we consider only the part of the current oscillating at frequencies $\Delta \omega \pm \omega_{m}$. Since this oscillating component is the replica of the modulated information, we call it $i_{S}(t)$. From Eqs. (1)-(4) with $\phi=\pi / 2, \dagger$ we find

$$
i_{D C}=\frac{\eta q}{2 h \nu} \int_{\Sigma} d \mathbf{r}\left[A_{\left.s^{2}(L, \mathbf{r})+A_{R^{2}}(L, \mathbf{r})\right]},\right.
$$

and

$$
\begin{array}{r}
i_{S}(t)=\frac{\eta q}{h \nu} 2 J_{1}(\delta) \sin \omega_{m} t \int_{\Sigma} d \mathbf{r} A_{R}(L, \mathbf{r}) A_{S}(L, \mathbf{r}) \\
\times \cos [\Delta \omega t+\Delta \phi(L, \mathbf{r})],
\end{array}
$$

where $J_{1}(\delta)$ is the first order Bessel function and $\Delta \phi(L, \mathbf{r})$ is the random phase difference between the information and the reference signal:

$$
\Delta \phi(L, \mathbf{r})=\phi_{S}(L, \mathbf{r})-\phi_{R}(L, \mathbf{r}) .
$$

Equations (5) and (6) are used below to derive expressions for the signal-to-noise ratio in heterodyne and video communication schemes.

\section{Signal-to-Noise Ratio for Superheterodyne Detection}

In a heterodyne detection scheme, the amplitude of the reference signal is constant over the plane of the detector $A_{R}(L, \mathrm{r})=A_{R}$ and the phase does not contain any fluctuations $\phi_{R}(L, \mathbf{r})=0$. In this case, $\Delta \phi(L, \mathbf{r})=$

$\dagger$ In the case of the video scheme, the optical bias $\phi=\pi / 2$, along with an output polarizer, is used to convert the phase modulation to amplitude modulation. In the heterodyne case, the choice of $\phi$ is immaterial and $\phi=\pi / 2$ is used for the sake of definiteness.
$\phi_{(1)}(L, \mathbf{r})$ where $\phi_{1}(L, \mathbf{r})$ is the phase of the modulated optical signal. The subscript (1) refers to heterodyne detection.

From Eq. (6) the signal part of the current is

$$
\begin{aligned}
i_{S}(t)=\frac{\eta q}{h_{\nu}} 2 J_{1}(\delta) A_{R} \sin \omega_{m} t \int_{\Sigma} d \mathbf{r} A_{(1)}(L, \mathbf{r}) \\
\quad \times \cos \left[\Delta \omega t+\phi_{(1)}(L, \mathbf{r})\right] ;
\end{aligned}
$$

$i_{S}(t)$, as expressed by Eq. (8), is the result of the beating of both sidebands of the signal beam at frequencies $\omega_{0}+\omega_{m}$ and $\omega_{0}-\omega_{m}$ with the reference beam at frequency $\omega_{0}-\Delta \omega$. In the remainder of this analysis, we use a slightly different expression for $i_{S}(t)$. We consider the current obtained by twice the contribution of a single sideband. It is expressed as

$$
\begin{array}{r}
i_{S}(t)=\frac{\eta q}{h \nu} 2 J_{1}(\delta) A_{R} \int_{\Sigma} d \mathbf{r} A_{(1)}(L, \mathbf{r}) \sin \left[\left(\Delta \omega+\omega_{m}\right)\right. \\
\left.\times t+\phi_{(1)}(L, \mathbf{r})\right] .
\end{array}
$$

It is simpler to relate $i_{S}(t)$ as expressed by Eq. (9) to known functions describing the turbulence, and the final result for $S / N$ is within a factor of one-half of the correct result which could be obtained from Eq. (8).

The signal power in the output current of the detector $S_{(1)}(t)$ is defined as $S_{(1)}(t)=i_{S}{ }^{2}(t)$. From Eq. (9),

$$
\begin{aligned}
& S_{(1)}(t)=\left(\frac{\eta q}{h \nu}\right)^{2} 4 J_{1}^{2}(\delta) A_{R^{2}} \int_{\Sigma} d \mathbf{r}_{1} \int_{\Sigma} d \mathbf{r}_{2} A_{(1)}\left(L, \mathbf{r}_{1}\right) A_{(l)}\left(L, \mathbf{r}_{2}\right) \\
& \times \sin \left[\left(\Delta \omega+\omega_{m}\right) t+\phi_{(1)}\left(L, \mathbf{r}_{1}\right)\right] \sin \left[\left(\Delta \omega+\omega_{m}\right) t+\phi_{(1)}\left(L, \mathbf{r}_{2}\right)\right] .
\end{aligned}
$$

The signal power $S_{(1)}$ averaged over a time large compared with $2 \pi /\left(\Delta \omega+\omega_{m}\right)$ is

$$
\begin{aligned}
S_{(1)}=\left(\frac{\eta q}{h \nu}\right)^{2} 2 J_{1}{ }^{2}(\delta) A_{R} \int_{\Sigma} & d \mathbf{r}_{1} \int_{\Sigma} d \mathbf{r}_{2} A_{(1)}\left(L, \mathbf{r}_{1}\right) A_{(1)}\left(L, \mathbf{r}_{2}\right) \\
& \times \cos \left[\phi_{(1)}\left(L, \mathbf{r}_{1}\right)-\phi_{(1)}\left(L, \mathbf{r}_{2}\right)\right],
\end{aligned}
$$

and the total statistically averaged power in the output current of the detector is expressed as

$$
\left\langle S_{(1)}\right\rangle=\left(\frac{\eta q}{h \nu}\right)^{2} 2 J_{1^{2}(\delta) A_{R^{2}}} \int_{\Sigma} \int_{\Sigma} d \mathbf{r}_{1} d \mathbf{r}_{2}\left\langle u_{(1)}\left(L, \mathbf{r}_{1}\right) u^{*}{ }_{(1)}\left(L, \mathbf{r}_{2}\right)\right\rangle,
$$

where the \langle\rangle denote ensemble averages and $u_{(1)}(L, \mathbf{r})$ is the wave function $u_{(1)}(L, \mathrm{r})=A_{(1)}(L, \mathrm{r}) \exp \left[i \phi_{(1)}-\right.$ $(L, r)]$ of the optical beam carrying the information. $\left\langle S_{(1)}\right\rangle$ is thus a function of the two-point correlation of the wave function in the plane of the detector.

In a detector where the largest source of noise is the shot noise generated by the local oscillator (reference) beam, the noise power is

$$
N_{(1)}=2 q B i_{D C}=\left(\eta q^{2} / h \nu\right) B A_{R^{2}\left(\pi D^{2} / 4\right),}
$$

where Eq. (5) has been used; $B$ is the bandwidth of the circuit following the detector and $D$ is the diameter of the detector. From Eqs. (12) and (13), the signal-tonoise ratio for a heterodyne detection system is

$$
\begin{array}{r}
(S / N)_{(1)}=\left\langle S_{(1)}\right\rangle / N_{(1)}=\frac{\eta}{h \nu B} 2 J_{1}{ }^{2}(\delta) \\
\quad \frac{1}{\left(\pi D^{2} / 4\right)} \int_{\Sigma} d \mathbf{r}_{1} \int_{\Sigma} d \mathbf{r}_{2} \\
\times\left\langle u_{(1)}\left(L, \mathbf{r}_{1}\right) u_{(1)}^{*}\left(L, \mathbf{r}_{2}\right)\right\rangle .
\end{array}
$$




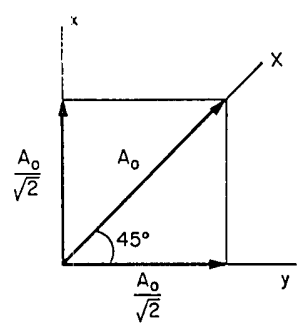

(a)

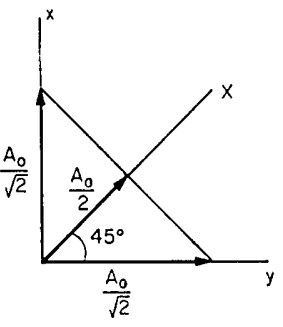

(b)
Fig. 1. Configuration of the polarizations in the case of a video communication scheme. The direction $X$ is the direction of the laser polarization and of the parallel polarizer.

\section{Signal-to-Noise Ratio for Video Communication}

In the video communication scheme, the amplitude and phase fluctuations of both the information signal and the reference signal are the same, since they follow the same atmospheric path:

$$
\phi_{R}(L, \mathbf{r})=\phi_{S}(L, \mathbf{r}) \text { or } \Delta \phi(L, \mathbf{r})=0 .
$$

We call $A_{(2)}(L, \mathbf{r})=A_{S}(L, \mathbf{r})=A_{R}(L, \mathbf{r})$ the common amplitude. The subscript (2) refers to the part of the beam carrying the information. With the help of Eqs. (6) and (15), the total statistically averaged signal power in the output current of the detector in a video communication scheme can be expressed as

$$
\left\langle S_{(2)}\right\rangle=\left(\frac{\eta q}{h \nu}\right)^{2} 2 J_{1}^{2}(\delta) \int_{\Sigma} \int_{\Sigma} d \mathbf{r}_{1} d \mathbf{r}_{2}\left\langle I_{(2)}\left(L, \mathbf{r}_{1}\right) I_{(2)}\left(L, \mathbf{r}_{2}\right)\right\rangle,
$$

where $I_{(2)}(L, \mathbf{r})=A_{(2)}{ }^{2}(L, \mathbf{r})$ is the intensity $\left(\mathrm{W} / \mathrm{m}^{2}\right)$ of the optical wave at the plane of the detector. $\left\langle I_{(2)}\right)^{-}$ $\left.\left(L, \mathbf{r}_{1}\right) I_{(2)}\left(L, \mathbf{r}_{2}\right)\right\rangle$ is the two-point correlation function of the intensity fluctuations in the plane of the detector.

In order to calculate $(S / N)_{(2)}$, we assume that the largest source of noise is the shot noise due to the optical signal itself. This condition corresponds to the ideal mode of operation of a video communication system. In a practical situation, a careful evaluation of all the parameters (areas of the transmitter and the receiver, length of propagation, wavelength, transmitted power, absorption coefficient of the atmosphere, sensitivity and noise equivalent power of the detector) is needed in order to determine whether this condition is satisfied..$^{2,3}$

According to Eq. (5), the statistically averaged signal shot noise power is

$$
\left\langle N_{(2)}\right\rangle=2 B \frac{\eta q^{2}}{h \nu} \int_{\Sigma} d \mathbf{r}\left\langle I_{(2)}(L, \mathbf{r})\right\rangle,
$$

and $(S / N)_{(2)}$ is given by the following expression:

$$
(S / N)_{(2)}=\frac{\eta}{h \nu B} J_{1^{2}(\delta)} \frac{\iint_{\Sigma} d \mathbf{r}_{1} d \mathbf{r}_{2}\left\langle I_{(2)}\left(L, \mathbf{r}_{1}\right) I_{(2)}\left(L, \mathbf{r}_{2}\right)\right\rangle}{\int_{\Sigma} d \mathbf{r}\left\langle I_{(2)}(L, \mathbf{r})\right\rangle} .
$$

The signal-to-noise ratio for video communication is thus a function of the correlation function of the intensity fluctuations in the plane of the detector.

\section{Comparison of the Performances}

We define the quantity $R=(S / N)_{(2)} /(S / N)_{(1)}$ which is a measure of the performance of a video communication system relative to that of a heterodyne communication system. According to Eqs. (14) and (18),

$$
R=\frac{1}{2} \frac{\left(\pi D^{2} / 4\right)}{\int_{\Sigma} d \mathbf{r} B_{u(2)}(L, \mathbf{r}, \mathbf{r})} \frac{\int_{\Sigma} \int_{\Sigma} d \mathbf{r}_{1} d \mathbf{r}_{2} B_{\mathbf{1}(2)}\left(L, \mathbf{r}_{1}, \mathbf{r}_{2}\right)}{\int_{\Sigma} \int_{\Sigma} d \mathbf{r}_{1} d \mathbf{r}_{2} B_{u(1)}\left(L, \mathbf{r}_{1}, \mathbf{r}_{2}\right)}
$$

where $B_{u}\left(L, \mathbf{r}_{1}, \mathbf{r}_{2}\right)=\left\langle u\left(L, \mathbf{r}_{1}\right) u^{*}\left(L, \mathbf{r}_{2}\right)\right\rangle$ is the correlation function of the wave function and $B_{1}\left(L, \mathbf{r}_{1}, \mathbf{r}_{2}\right)=\left\langle I\left(L, \mathbf{r}_{1}\right)-\right.$ $\left.I\left(L, \mathrm{r}_{2}\right)\right\rangle$ is the correlation function of the intensity. The subscripts (1) and (2) refer to the part of the optical beam carrying the information in the heterodyne and video communication schemes, respectively.

In the absence of turbulence $R=\frac{1}{2}\left(A_{(2)}{ }^{2} / A_{(1)}{ }^{2}\right.$, we assume that the same laser optical beam with amplitude $A_{0}$ at the plane of the detector is used as a carrier for both communication schemes. In the case of heterodyne detection, the phase of the total laser electric vector is modulated; then $A_{(1)}=A_{0}$. In the case of video communication, the laser polarization is chosen to have equal components along two perpendicular directions. One polarization component is used as the information signal, the other as the transmitted reference signal; then only the amplitude $A_{0} /(2)^{\frac{1}{2}}$ is modulated [see Fig. 1(a)]. A polarizer is used at the detector to mix the information and the reference signals. If the polarizer is parallel to the laser polarization, the amplitude of the modulated optical signal in this direction is $A_{(2)}=\left[A_{0} /(2)^{\frac{1}{2}}\right]\left[1 /(2)^{\frac{1}{2}}\right]=\left(A_{0} / 2\right)$ [see Fig. 1(b) $]$. Then in the absence of turbulence, $R=\frac{1}{8}$; i.e., the $S / N$ for the superheterodyne system is larger by a factor of eight than that of a video system.

In order to calculate $R$ in the presence of atmospheric turbulence, we use the results of a theoretical analysis of optical wave propagation through a random medium which is reported elsewhere. ${ }^{4}$

Assuming that the refractive index fluctuations are a homogeneous ${ }^{5}$ gaussian random process, the wave function correlation function is expressed in terms of the

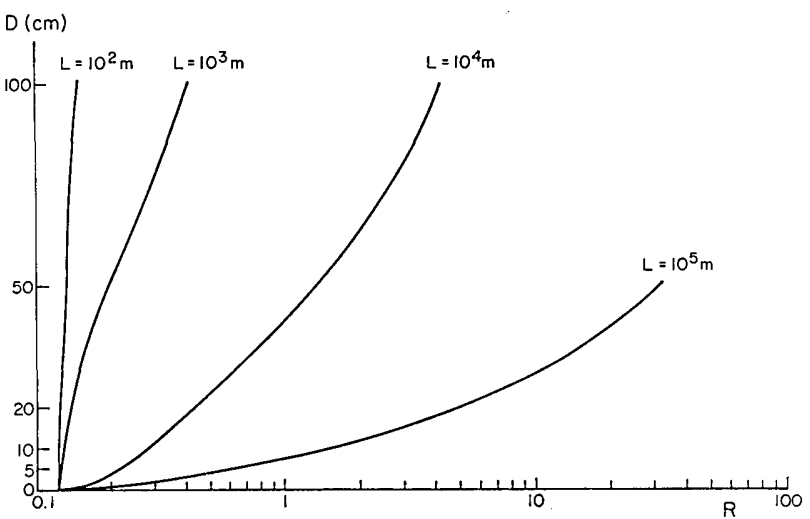

Fig. 2. $R$ vs $D$ for a weak turbulence $C_{n}=10^{-8} \mathrm{~m}^{-1 / 3}$ at $\lambda=1 \mu$. 


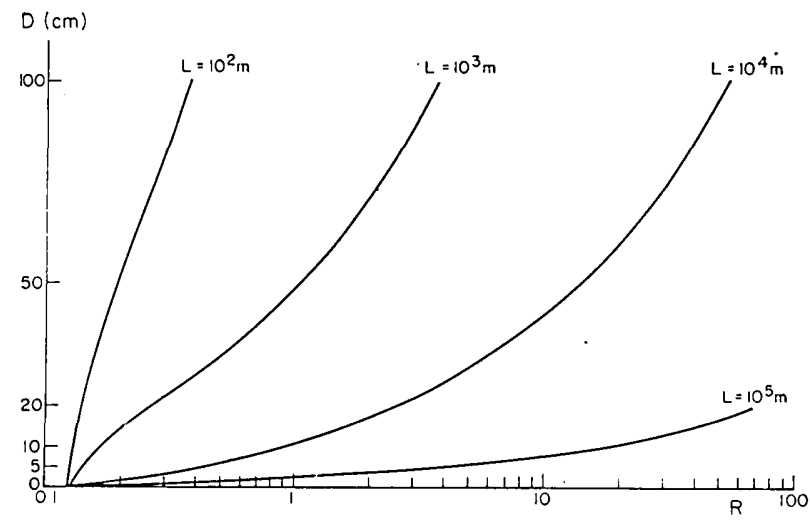

Fig. 3. $R$ vs $D$ for an intermediate turbulence $C_{n}=3 \times 10^{-8}$ $\mathrm{m}^{-1 / 3}$ at $\lambda=1 \mu$.

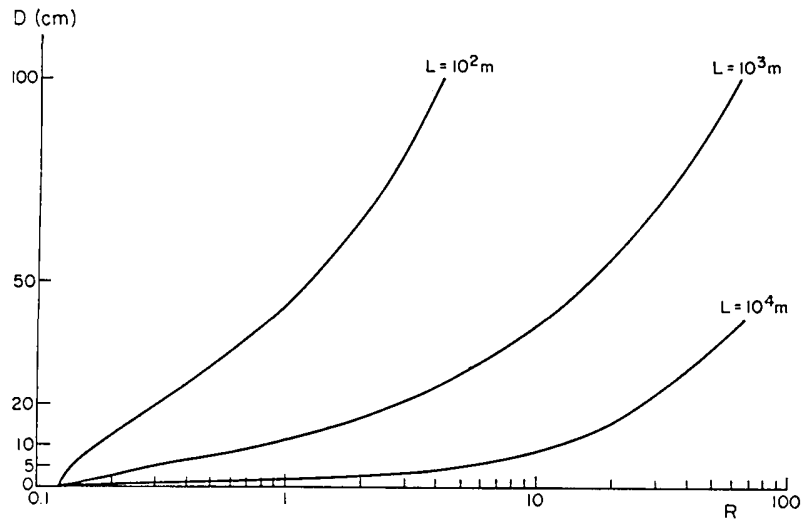

Fig. 4. $R$ vs $D$ for a strong turbulence $C_{n}=10^{-7} \mathrm{~m}^{-1 / 3}$ at $\lambda=$ $1 \mu$.

correlation function of the index of refraction $B_{n}(\alpha)$ in the following way:

$$
\begin{aligned}
& \left.B_{u(1)}\left(L, \mathbf{r}_{1}, \mathbf{r}_{2}\right)=B_{u(1)}(L, \rho)=A_{(1)}\right)^{2} \exp \left[-2 k^{2} L\right. \\
& \left.\times\left(\int_{0}^{L_{0}} B_{n}(\alpha) d \alpha-\int_{0}^{\left(L_{0^{2}}-\rho^{2}\right)^{\frac{3}{2}}} B_{n}\left(\alpha^{2}+\rho^{2}\right)^{\frac{2}{2}}\right) d \alpha\right] ;
\end{aligned}
$$

$L_{0}$ is the outer scale of the turbulence, and $\rho=\left|\mathrm{r}_{1}-\mathrm{r}_{2}\right|$. No theory to date has proven successful in finding an exact analytical expression for the intensity correlation function $B_{\mathrm{I}}\left(L, \mathbf{r}_{1}, \mathbf{r}_{2}\right)$ which is applicable beyond the limits of validity of the Rytov approximation ${ }^{4}$ (long propagation paths, strong turbulences). For the purpose of a numerical application, we choose an expression for $B_{I}$ which fits the recent experimental data of Gracheva for small and large $\sigma_{1}(L, 0)^{4,6}$ as defined by Eq. (22).

$$
B_{I(2)}\left(L, \mathbf{r}_{1}, \mathbf{r}_{2}\right)=B_{I(2)}(L, \rho)=A_{(2)^{4}}\left[2-\frac{1}{1+\sigma_{1}{ }^{2}(L, \rho)}\right],
$$

where

$\sigma_{1}^{2}(L, \rho)=8 \pi^{2} k^{2} L \int_{0} J_{0}(K \rho)$

$$
\times\left(1-\frac{k}{K^{2} L} \sin \frac{K^{2} L}{k}\right) \phi_{n}(K) K d K
$$

$\phi_{n}(K)$ is the three-dimensional Fourier transform of the refractive index correlation function. In deriving Eq. (21), we have used the assumption which is supported by experimental evidence, ${ }^{6-9}$ that the amplitude of an optical wave propagating through atmospheric turbulence has a log-normal probability distribution function.

With the help of Eqs. (20)-(22) and (19), we have performed a numerical calculation of $R$ using the Kolmogoroff-Obukhor model of turbulence: ${ }^{10}$

$$
\phi_{n}(K)=\left\{\begin{array}{cl}
0.033 C_{n}^{2} K^{-\frac{11}{3}} & \text { for } K<K_{m}=5.48 / l_{0} \\
0 & \text { for } K>K_{m}
\end{array}\right.
$$

and have expressed $R$ in terms of the diameter of the detector $D$, the wavelength $\lambda$, the communication distance $L$, and the turbulence strength as represented by the structure constant $C_{n}$. The results are presented in Figs. 2-6.

In Fig. 2, the performance criterion $R$ is plotted as a function of the detector diameter $D$ for a communication link at $1 \mu$ propagating up to a distance of $100 \mathrm{~km}$ through a weak turbulence $\left(C_{n}=10^{-8} \mathrm{~m}^{-\frac{1}{3}}\right) . \quad R$ vs $D$ plots for the same communication system in intermedi-

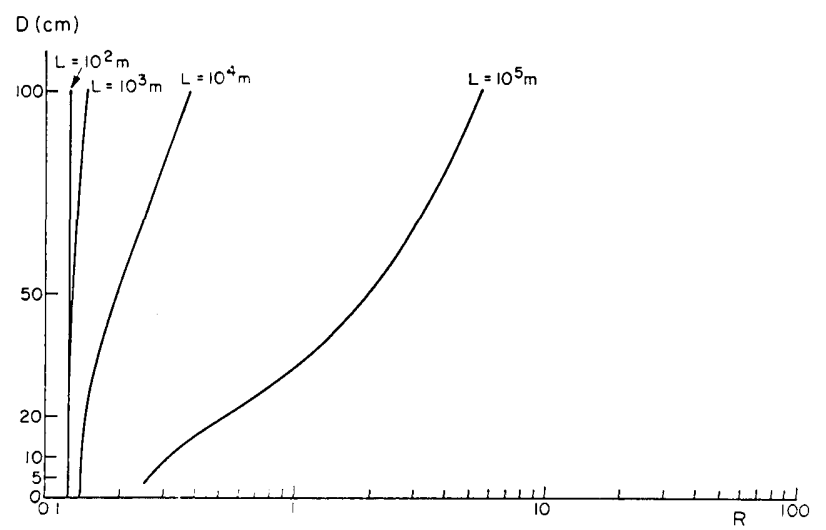

Fig. 5. $R$ vs $D$ for an intermediate turbulence $C_{n}=3 \times 10^{-8}$ $\mathrm{m}^{-1 / 3}$ at $\lambda=10 \mu$.

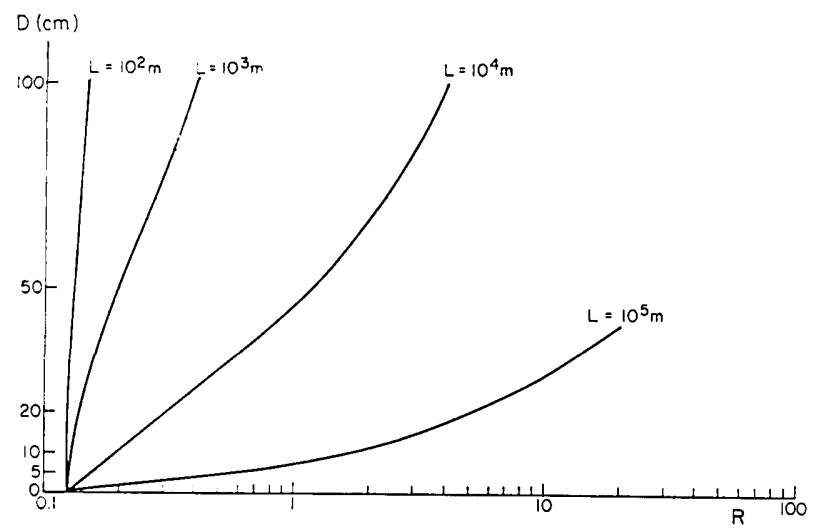

Fig. 6. $R$ vs $D$ for a strong turbulence $C_{n}=10^{-7} \mathrm{~m}^{-1 / 3}$ at $\lambda=$ $10 \mu$. 
ate $\left(C_{n}=3 \times 10^{-8} \mathrm{~m}^{-\frac{1}{3}}\right)$ and strong $\left(C_{n}=10^{-7}\right.$ $\left.m^{-\frac{1}{2}}\right)$ turbulences are given in Figs. 3 and 4 , respectively. It is found that $R$ is an increasing function of the detector diameter of the propagation distance and of the turbulence strength. For a propagation distance of $10 \mathrm{~km}$ at $1 \mu$, as an example, the video communication scheme has a larger signal-to-noise ratio than the heterodyne detection scheme $(R>1)$ when $D>40 \mathrm{~cm}, D>10$ $\mathrm{cm}$, and $D>2 \mathrm{~cm}$ in weak, intermediate, and strong turbulences, respectively. A video communication scheme would perform even better for longer propagation distances, stronger turbulences, and shorter wavelengths. This is due to the cancellation of the phase fluctuations between the reference and the signal parts of the beam.

In Figs. 5 and 6, we have represented $R$ vs $D$ for a communication link at a wavelength of $10 \mu$ under intermediate and strong turbulences. In this case, $R$ increases more slowly with $D, L$, and $C_{n}$ than for $\lambda=1 \mu$. It is only for long communication lengths under strong turbulence $\left(L=100 \mathrm{~km}, C_{n}=10^{-7} \mathrm{~m}^{-\frac{1}{3}}\right)$ that a video system would be preferable to a superheterodyne detection system. This is due to the fact that the correlation function $B_{u}(L, \rho)$, as expressed by Eq. (20), varies as $e^{-1 / \lambda^{2}}$ and therefore decreases more slowly with a longer wavelength. The phase fluctuations are less important at $10 \mu$ than at $1 \mu$.

\section{Conclusion}

We have calculated the $(S / N)$ for (1) a superheterodyne and (2) a video optical communication system, involving propagation through the atmospheric turbulence. It has been found that for long propagation distances in a weak turbulence, or for a propagation distance of a few kilometers in an intermediate turbulence, scheme (2) operating at $1 \mu$ has a larger signal- to-noise ratio than scheme (1). However, a heterodyne system operating at $10 \mu$ is less sensitive to the random phase fluctuations introduced by the atmosphere than a system at $1 \mu$.

As far as the effects of atmospheric turbulence are concerned, at $1 \mu$ and smaller wavelengths a video system is preferable to a superheterodyne system, while at $10 \mu$ and longer wavelengths, the inverse is true. These theoretical results are in agreement with some recent experimental results. ${ }^{11}$

The authors wish to thank the Army Research Office-Durham, and the NASA Electronic Research Center, for the support of this work.

\section{References}

1. R. F. Lucy, K. Lang, C. J. Peters, and K. Duval, Appl. Opt. 6, 1333 (1967).

2. J. R. Kerr, Proc. IEEE 55, 1686 (1967).

3. D. L. Fried and J. B. Seidman, Appl. Opt. 6, 245 (1967).

4. J. P. Laussade and A. Yariv, "A Theoretical Study of Optical Wave Propagation through Random Atmospheric Turbulence," to be published.

5. A. M. Yaglom, An Introduction to the Theory of Stationary Random Functions (Prentice-Hall, Inc., Princeton, 1962).

6. M. E. Gracheva, Izv. VUZ Radiofiz. 8, 775 (1967).

7. M. E. Gracheva and A. S. Gurvich, Izv. VUZ Radiofiz. 8, 717 (1965).

8. G. R. Ochs, R. R. Bergman, and J. R. Snyder, "Laser Beam Scintillations over Horizontal Paths from 5.5 to 145 Kilometers," to be published.

9. G. R. Ochs and R. S. Lawrence, "Saturation of Laser Beam Scintillation under Conditions of Strong Atmospheric Turbulence," to be published.

10. V. I. Tatarski, Wave Propagation in a Turbulent Medium (McGraw-Hill Book Company, Inc., New York, 1961), p. 110.

11. R. F. Lucy and K. Lang, Appl. Opt. 7, 1965 (1968).

\section{Applied
Optics BOOKS}

\begin{abstract}
Books for review should be submitted to the Editorial Consultant, P. R. Wakeling, WINC, 1613 Nineteenth Street, $N$. W., Washington, D.C. 20009
\end{abstract}

Radiation in the Atmosphere. By $K . Y_{A}$. Kondratyev. Academic Press, New York, 1969 . 912 pp. $\quad \$ 39.00$.

This book is the twelfth volume of the International Geophysics Series edited by J. van Mieghem of Belgium. These twelve titles have now been published since 1959 (and two others are listed as in preparation), all by distinguished names in the meteorological community. The only other one in this series so far that has substantially concerned optics and spectroscopy was the Physics of the Aurora and Airglow by J. W. Chamberlain, published in 1961 .

The volume is a thorough and up-to-date treatment of radiative transfer in the atmosphere and the other processes of atmospheric thermal radiation that largely control our climate and weather.
The book is organized into eleven chapters. After an initial chapter on the concepts and definitions of thermal radiation and radiative transfer, there is a chapter on methods of actinometric measurement, including descriptions of all of the standard meteorological instruments for measuring duration and amount of sunshine: pyrheliometers, actinometers, and pyranometers. Then follows a detailed discussion of the processes of absorption of radiation by the principal atmospheric constituents; and then a chapter on aerosol scattering. Following these are four chapters on ideal and real atmospheres, including such topics as slant path transmission, the albedo of terrain and clouds, and diffuse radiation in the atmosphere. All these chapters, which examine physical processes in detail, occupy the first half of the book. The other half considers global effects of radiative transfer and its influence on weather and climate. This latter half is more meteorologically oriented, treating radiation charts as a function of season and latitude and discussing radiative exchange from troposphere through mesophere to stratosphere.

Several sections of this book are updated versions of material originally treated in several monographs on specific topics that Kondratyev has published (in Russian) in recent years. One or 
two of these were also later translated into English, but suffer from the infelicities that result when the translator does not know exactly what the author had in mind. Kondratyev himself has an excellent command of English, and this book has no such shortcomings; he wrote this book directly in English. The style is lucid and readable (with the mathematical derivations handled much in the form of a lecture: "Let us now follow the technique of this author. Remember that he has assumed local thermodynamic equilibrium..."). Techniques are illustrated with charts and graphs of real data, and Kondratyev comments on the strong points or weaknesses of various methods. The number of references is prodigious: a hundred or two for each chapter, and when he includes a reference he has read this reference and comments on it specifically or uses data from it. In the back of the book there is an additional bibliography of some 440 papers (all dated 1966, 1967, or 1968).

Atmospheric radiation is a lively and active field with physicists, meteorologists, and astronomers all contributing to it, and sometimes even speaking to each other. The active workers are about $40 \%$ American, $40 \%$ Russian, and $20 \%$ everybody else, and Kondratyev appears to follow and understand the work of all of these colleagues and has here organized and interpreted their results in a form palatable to us all.

John N. HowaRD

The Invention of the Meteorological Instruments. By W. E. Knowles Middleton. The Johns Hopkins Press, Baltimore, 1969. 362 pp. $\$ 12.00$.

W. E. Knowles Middleton is a distinguished authority in three separate but related fields. One of his principal specialities has been in atmospheric optics and visibility, and he has long been active both in optics and in meteorology. In 1959 he was the Ives Medalist of the Optical Society of America, and his Ives Medal address in the February 1960 issue of J. Opt. Soc. Amer. summarizes some of these interests and is accompanied by a good bibliography of his publications to that date. His third interest has been in the history of meteorology, and in recent years he has published scholarly books such as on the History of the Thermometer, the History of the Barometer, the History of the Theories of Rain, and now the volume under review.

From his earliest beginnings, man, when not engaged in slaughtering his neighbors, has been primarily interested in his material comfort and well being, and in taming the world of plants and animals to his will. He has also had a deep interest and curiosity about his natural environment, and astronomy is perhaps the oldest science. Even though mankind is much more tangibly involved with weather and climate than with the more distant stars and planets, the science of meteorology is much more recent than most of the sciences, and systematic, coordinated efforts to understand, to measure, or to predict the weather have occurred for the most part all within the last hundred years. Attempts to measure rain were begun in India as early as the Fourth Century B.C., and windvanes were known to the Greeks in 100 B.C., but the real beginnings of meteorological instruments belong to early seventeenth century Italy with the air thermoscope, the anemometer, the barometer of Torricelli and the sealed liquid-in-glass thermometer (much as we know it today). Later in that century the initiative in such matters passed to northern Europe, where many of these instruments were improved and refined. Physicists, chemists, and astronomers such as Torricelli, Hooke, Bernouilli, Boyle, Pascal, Descartes, Dalton and Romer all devised meterological instruments, and by the year 1800 recording instruments with clockwork and moving charts had been constructed and many of the scales were standardized.
Optics played only an insignificant role among such instruments until the mid 1800's. With the photography of Fox Talbot and Daguerre came sunshine recorders (although Kircher had discussed such an instrument in 1646). George Stokes devised a standard instrument for the Meteorological Office. Optical techniques were also used to measure the height and motions of clouds, and the use of searchlight beam as a ceilometer dates from about 1872. The optical tracking of balloons and kites to measure winds aloft dates from before 1800 .

Middleton ends his account of meteorological instruments with the balloon-borne radiosondes developed during and just after World War II. The newer and later optical and spectroscopic techniques to measure total ozone and ozone profiles by the umkehr method, the use of satellite-borne radiometers and photometers to measure cloud cover, vertical temperature profile, humidity profile, or searchlight and laser probing techniques to measure aerosols are purposefully not covered: he leaves these to later historians: he has here dealt with the beginnings of the standard instruments.

Middleton brings to his writing a combination of lucid, logical and interesting style, and careful scholarship. Facts are footnoted and documented, and many obscure works in ancient and medieval tongues are translated and interpreted for the reader. The diagrams and drawings, mostly from the original papers, are beautifully done, and make the volume a work of art as well as a scholarly text. This book represents a great amount of careful hard work by the author, and everyone interested in how we measure our physical environment will profit from it.

JoHN N. Howard

Light, Colour and Vision. By Yves Le Grand. Translated by R. W. G. Hunt and F. R. W. Hunt. Chapman and Hall, Ltd, London, 1968. $564 \mathrm{pp}$. $\$ 11.25$.

All too frequently a dust jacket contains laudatory comments which the book does not warrant. However, in this case the praise is richly deserved. Yves Le Grand is the author of a three-volume work entitled Traité d'optique physiologique of which the first volume, La dioptrique de l'oeil et sa correction, is still untranslated into English and the third volume, L'epsace visull has been published in an English translation entitled Form and Space Vision. Light, Colour and Vision is the English translation of the second volume. The first edition was translated and published in 1958. This second edition contains a host of new material with references to the literature as recent as 1966, and the final four chapters on the photochemistry of the retina, and electrophysiology of the retina, theories of color vision, and theories of the thresholds have been extensively rewritten to include recent developments in the field.

The book is written, as the author admits, from the point of view of a physicist, and its is perhaps because of this concentration on a particular overall viewpoint that the book achieves a cohesive whole. There are two major sections within the book. The first is entitled Experimental Facts and comprises fifteen chapters including the concept of radiant energy, the sources of radiation, a description of the human eye, photometry, luminous efficiency, colorimetry, color vision and its anomalies, threshold measurements, time effects, and spatial interactions. The second section-Theories of Vision-comprises five chapters starting with the anatomy of the retina followed by the four rewritten chapters. This latter section draws on aspects of the fields of anatomy, physiology, and psychology necessary for an understanding of the concepts that are presented.

One expects a book of this type, which purports to become a standard work in this field for both student and practicing scien- 